This article is licensed under the Creative Commons Attribution-NonCommercial 4.0 International License (CC BY-NC) (http://www.karger.com/Services/OpenAccessLicense). Usage and distribution for commercial purposes requires written permission.

\title{
Tuberculosis of the Hand with Isolated Hamate Involvement: An Unusual Presentation
}

\author{
Hari Kishan Abhishek Kashyap Shreesh Kadur Anurag Soni \\ Sandeep Sehrawat
}

Department of Orthopaedics, Maulana Azad Medical College and Lok Nayak Hospital, New Delhi, India

\section{Keywords}

Carpal bone $\cdot$ GenXpert · Hamatum

\section{Abstract}

Osteoarticular tuberculosis accounts for $1-5 \%$ of all tuberculosis cases, rarely involving the wrist or hand. Wrist and hand tuberculosis usually presents with multiple carpal bone involvement with impaired wrist and hand function. Isolated involvement of the carpal bone, particularly the hamate, is very rare. We report a 12-year-old female child with isolated hamate bone involvement without any limitation of wrist function. The patient was managed conservatively on antitubercular drugs. Such a case has not been reported in the past. 


\section{Case Reports in Orthopedic Research}

Case Rep Orthop Res 2019;2:61-68

DOI: $10.1159 / 000502733$

Kishan et al:: Tuberculosis of the Hand with Isolated Hamate Involvement: An Unusua Presentation

\section{Introduction}

Tuberculosis (TB) of the wrist and hand is a rare entity. It comprises $2-4 \%$ of all musculoskeletal TB cases [1]. Common sites of primary osseous focus are the os capitatum or distal end of the radius [2]. Very few cases have been reported of isolated involvement of a single bone or joint in the wrist or hand [3-8]. Isolated involvement of the hamate, in particular, is even rarer. We report a 12-year-old female child with tuberculous osteitis of the hamatum without any functional limitation of the hand or wrist.

\section{Case Report}

A 12-year-old female child presented to our outpatient department with the complaint of swelling and pain over the ulnar aspect of the hand without any functional limitation of the hand or wrist over the last 2 months. The patient also had intermittent low-grade fever during this period. It was also associated with weight loss and anorexia. On local examination, a discharging sinus was identified on the dorsal aspect of the hand near the base of the 5th metacarpal bone. A small superficial soft swelling was also identified distal to the discharging sinus. There was no swelling in the ganglionic areas of the wrist. The wrist's range of motion was comparable to the normal side with normal fingers' range of motion (Fig. 1a, b). There was no distal neurological or vascular deficit in the hand. Erythrocyte sedimentation rate was $45 \mathrm{U} / \mathrm{L}$ (normal values: 0-10 U/L for males; 0-20 U/L for females) and total leucocyte count was $6,500 / \mathrm{mm}^{3}$ (normal values: $4,000-11,000 / \mathrm{mm}^{3}$ ). C-reactive protein $(2 \mathrm{mg} / \mathrm{L}$ ) was done but was normal. X-rays of the hand and wrist (Fig. 1c) showed multiple lytic lesions in the hamate bone. A chest radiograph was also obtained but was normal without any hilar or apical opacity. A contrast-enhanced magnetic resonance imaging (MRI) was performed which showed altered signal intensity in the hamate bone, hypointense on T1 and hyperintense on T2, with cavity formation showing intense postcontrast enhancement (Fig. 2a, b). An enhancing sinus tract was also seen extending from the cavitation in the body of the hamate and traversing between the extensor tendons to the subcutaneous plane to the skin surface.

Around 3-4 mL of fluid was collected from the sinus after applying pressure over the swelling and was sent for Gram stain and Ziehl-Neelsen (ZN) stain, GeneXpert test, bacterial solid culture (Löwenstein-Jensen medium), and liquid culture (mycobacterium growth indicator tube [MGIT]) for TB. Fine needle aspiration was done from the swelling using a 16-gauge needle and sent for analysis. Gram stain and ZN stain were negative. GenXpert test was positive for mycobacteria and sensitive to rifampicin. Histologic evaluation from fine needle aspirate also suggested granulomatous inflammation with caseous necrosis in a background of mononuclear giant cells. Bacterial culture on solid medium showed no growth. MGIT culture suggested positive culture for Mycobacterium tuberculosis sensitive to isoniazid and rifampicin.

The patient was started on first-line antitubercular drugs as per World Health Organization (WHO) guidelines [9], and the wrist was splinted in functional position using a belowelbow slab. The slab was removed after 6 weeks and the wrist was mobilized. In a period of 6 


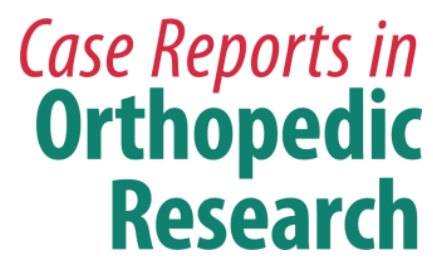

Case Rep Orthop Res 2019;2:61-68

Kishan et al.: Tuberculosis of the Hand with Isolated Hamate Involvement: An Unusual Presentation

months, the swelling decreased, the discharging sinus healed, and the patient reported improvement in constitutional symptoms (Fig. 3).

\section{Discussion}

India accounts for one-fourth of the global cases of TB, adding $\sim 2.8$ million cases annually [10]. TB commonly involves the lungs, but $10 \%$ of cases manifest as extrapulmonary TB. According to a report from the Revised National TB Control Program, extrapulmonary TB accounts for $20 \%$ of the total TB cases in India [10]. Osteoarticular TB accounts for $1-5 \%$ of the total TB cases and $10-18 \%$ of the extrapulmonary cases [11].

TB of both the wrist and hand is very rare and represents $2-4 \%$ of musculoskeletal TB [1]. Wrist joint TB usually presents as a painless, progressive swelling through tendons without any other systemic sign or symptom. TB of the wrist may start in the synovium or rarely in the carpus. The disease in the synovium disseminates to involve the whole carpus. Common sites of primary osseous focus are the os capitatum or distal end of the radius. Secondary concomitant involvement of the flexor and extensor sheaths may also occur. Abscess and sinus formation and regional lymph node enlargement are common [2].

Provisional diagnosis of musculoskeletal TB of the wrist and hand is usually clinic-radiological. The Phemister triad describes some common radiographic findings in osteoarticular TB, which includes juxta-articular osteopenia, bony erosions, and reduced joint space [12]. Tuberculous lesions are usually lytic and lack sclerosis, sequestra, and periosteal reactions. Sclerosis is more commonly seen in adults than in children [13]. MRI is the imaging modality of choice for early detection of musculoskeletal TB. Synovial proliferation due to tuberculous arthritis is typically hypointense on T2-weighted images and enhances vividly after administration of intravenous gadolinium contrast. Associated bone marrow edema, osteomyelitis, and soft tissue abnormalities, such as myositis, cellulitis, para-articular abscess formation, tenosynovitis, bursitis, and skin ulceration/sinus tract formation, may be seen. Sinus tracts are characterized by linear high signal intensity on T2-weighted images with marginal "tramtrack enhancement" with gadolinium [14].

Conventional light microscopy of ZN-stained smears prepared directly from specimens is the most widely available test for the diagnosis of TB. The new, rapid, and fully automated Xpert MTB/RIF test is a real-time cartridge-based automated DNA amplification test [15]. The GeneXpert test has $100 \%$ sensitivity and $91.6 \%$ specificity for extrapulmonary samples [16]. The WHO also recommended the GeneXpert test for the diagnosis of musculoskeletal TB in 1993 [17]. Culture and biopsy remain the gold standard for diagnosing TB.

Very few cases of isolated bone or joint involvement in wrist and hand TB have been reported (Table 1). Kushwaha et al. [3] reported a case of isolated involvement of the 3rd metacarpal bone in 2008. Kotwal and Khan [18] reported 32 cases of wrist and hand TB in 2009, 12 patients with bony and 20 with primarily soft-tissue involvement. The phalanges were primarily involved in 4 cases, the metacarpals in 6 ( 5 th metacarpal being the most common), and capitate in 2. Pain and swelling are the most common presenting features, followed by discharging sinuses. In 2014, Prakash [4] reported isolated involvement of the capitate. In 2015, Sbai et al. [5] and Goel et al. [6] reported isolated scapholunate and hamate involvement. 
Prakash and Mehtani [7] reported isolated involvement of the scaphoid bone. In 2018, Grenho et al. [8] also reported isolated involvement of the capitate with carpal synovitis. All the abovementioned cases had restriction of wrist and hand function with involvement of the adjacent intercarpal joints.

Our case was unique in multiple aspects. It presented in a pediatric age group, which is rare. There was isolated involvement of the hamate bone with a communicating sinus. Isolated carpal bone involvement has been described in the literature (Table 1). However, our case has a few peculiar findings, such as no restriction of wrist motion on presentation, intact articular surfaces of the hamate, and no intercarpal arthritis. Radiologically, the articular surfaces of the hamate were completely normal. Even on MRI, there was no synovitis in nearby intercarpal joints. There was limited tenosynovitis of the extensor tendons. The disease was restricted to the hamate probably because of the sinus itself. Since the purulent content had an exit tract, it did not go to adjacent structures.

Musculoskeletal TB is usually a paucibacillary disease, and culture may not always be positive in these patients. However, our case had both MGIT culture and GeneXpert test positive, which confirms the diagnosis. Also, the needle aspirate was suggestive of granulomatous infection indicative of tuberculous infection.

The patient was started on multidrug antitubercular therapy (isoniazid, rifampicin, pyrazinamide, and ethambutol) with improvement of signs and symptoms over a period of 6 weeks. She was continued on antitubercular treatment for a period of 12 months. At 12 months, the patient was completely asymptomatic with normal wrist function and normal range of motion.

\section{Conclusion}

The diagnosis of isolated carpal or metacarpal bone TB requires a high index of suspicion, especially when functional limitation is not significant. Localized tenderness, a discharging sinus, constitutional symptoms, and chronicity of symptoms suggest the possibility of TB of carpal bones even with normal wrist function. Plain radiographs and MRI may help in making the diagnosis. For confirmation of the diagnosis, GeneXpert, biopsy, and culture should be considered. GeneXpert provides swift results, helping in the early institution of treatment in contrast to biopsy and culture, which take a longer time to give results (biopsy takes $\sim 7$ days and culture takes $\sim 4$ weeks). Early institution of multidrug therapy may result in complete healing. However, limitation of function may depend on early diagnosis, initial function, bone and tissue destruction at presentation, and any surgical intervention done during the course of treatment.

\section{Statement of Ethics}

A written informed consent was obtained from both parents of the child to publish the case and images pertaining to it. A written informed consent was also obtained for the needle biopsy conducted for the study. 


\section{Disclosure Statement}

The authors declare that they have no conflicts of interest.

\section{Author Contributions}

Each author contributed equally to the preparation of the manuscript.

\section{References}

1 Benkeddache Y, Sidhoum SE, Derridj A. Les différents aspects des tuberculoses de la main: à propos d'une série de 45 cas. Ann Chir Main. 1988;7(2):166-75.

2 Tuli SM. Tuberculosis of the Skeletal System. 3rd ed. New Delhi: Jaypee Brothers Medical Publishers (P) Ltd.; 2004. pp. 153-8. https://doi.org/10.5005/jp/books/10993.

3 Kushwaha RA, Kant S, Verma SK, Sanjay, Mehra S. Isolated metacarpal bone tuberculosis-a case report. Lung India. 2008 Jan;25(1):17-9.

4 Prakash J. Tuberculosis of capitate bone in a skeletally immature patient: a case report. Malays Orthop J. 2014 Mar;8(1):72-4.

5 Sbai MA, Benzarti S, Bouzaidi K, Sbei F, Maalla R. A rare localization of tuberculosis of the wrist: the scapholunate joint. Int J Mycobacteriol. 2015 Jun;4(2):161-4.

6 Goel A, Sabat D, Sambharia AK. Tuberculosis of wrist joint in an 8-year-old child presenting as hamate osteitis: A rare case report. J Clin Orthop Trauma. 2015 Dec;6(4):293-5.

7 Prakash J, Mehtani A. Isolated tuberculosis of scaphoid in the skeletally immature: a rare cause of chronic wrist pain. BMJ Case Rep. 2015 May;2015.

8 Grenho A, Arcângelo J, Jordão P, Gouveia C. Carpal synovitis with capitate bone tuberculosis in a child. BMJ Case Rep. 2018 Mar;2018.

9 World Health Organization (WHO). Essential first-line antituberculosis drugs. In: WHO, editors. Treatment of Tuberculosis. Guidelines. 4th ed. Geneva: WHO; 2010. pp. 103-114.

10 Ministry of Health and Family Welfare. Central Tuberculosis Division, Government of India. Available from: http://www.tbcindia.nic.in/WriteReadData/TB\%20India\%202017.pdf

11 Ruiz G, García Rodríguez J, Güerri ML, González A. Osteoarticular tuberculosis in a general hospital during the last decade. Clin Microbiol Infect. 2003 Sep;9(9):919-23.

12 Phemister DB. The effect of pressure on articular surfaces in pyogenic and tuberculous arthritides and its bearing on treatment. Ann Surg. 1924 Oct;80(4):481-500.

13 Morris BS, Varma R, Garg A, Awasthi M, Maheshwari M. Multifocal musculoskeletal tuberculosis in children: appearances on computed tomography. Skeletal Radiol. 2002 Jan;31(1):1-8.

14 Danchaivijitr N, Temram S, Thepmongkhol K, Chiewvit P. Diagnostic accuracy of MR imaging in tuberculous spondylitis. J Med Assoc Thai. 2007 Aug;90(8):1581-9.

15 Cepheid International. Xpert MTB/RIF: Two-hour detection of MTB and rifampin-resistance mutations. Cepheid International, 2011. Available from: https://www.cepheid.com/en/cepheid-solutions/clinical-ivdtests/critical-infectious-diseases/xpert-mtb-rif

16 Ioannidis P, Papaventsis D, Karabela S, Nikolaou S, Panagi M, Raftopoulou E, et al. Cepheid GeneXpert MTB/RIF assay for Mycobacterium tuberculosis detection and rifampin resistance identification in patients with substantial clinical indications of tuberculosis and smear-negative microscopy results. J Clin Microbiol. 2011 Aug;49(8):3068-70.

17 World Health Organization. Automated Real Time Nucleic Acid Amplification Technology for Rapid and Simultaneous Detection of Tuberculosis and Rifampicin Resistance: Xpert MTB/RIF Assay for the Diagnosis of Pulmonary and Extrapulmonary TB in Adults and Children: Policy Update. Geneva: World Health Organization; 2013.

18 Kotwal PP, Khan SA. Tuberculosis of the hand: clinical presentation and functional outcome in 32 patients. J Bone Joint Surg Br. 2009 Aug;91(8):1054-7. 


\section{Case Reports in Orthopedic Research}

Case Rep Orthop Res 2019;2:61-68

DOI: $10.1159 / 00050273$

(c) 2019 The Author(s). Published by S. Karger AG, Basel www.karger.com/cio

Kishan et al.: Tuberculosis of the Hand with Isolated Hamate Involvement: An Unusual Presentation
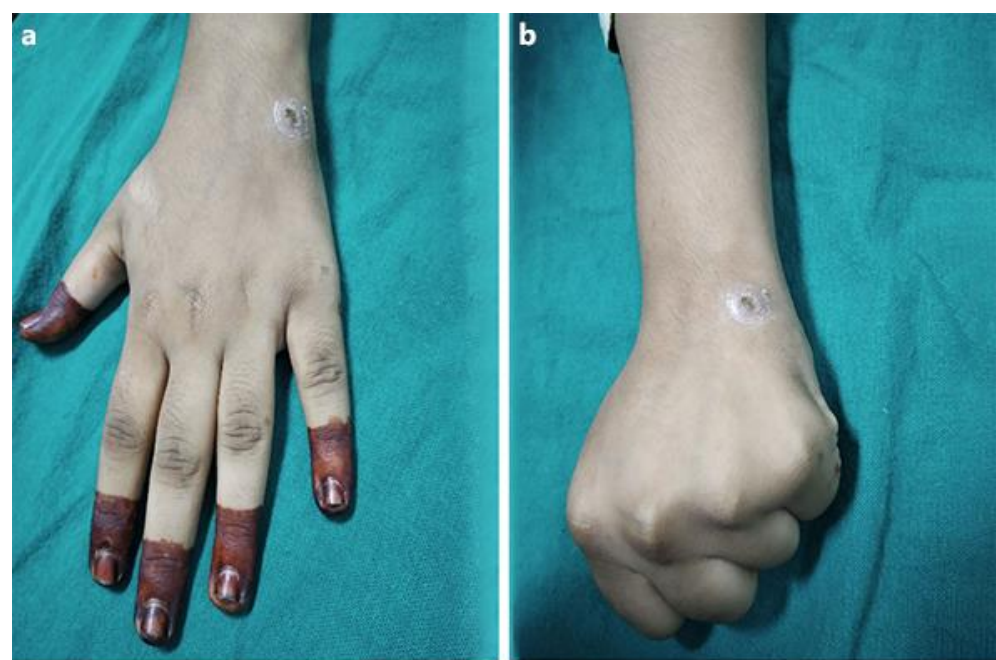

C

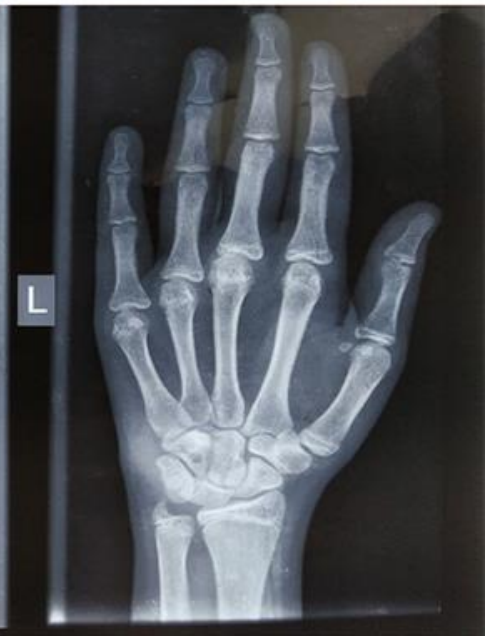

Fig. 1. a, b Clinical pictures showing sinus at the ulnar border of the dorsum of the wrist on presentation. c AP and oblique views of the hand showing lytic lesion in the hamate bone. 


\section{Case Reports in Orthopedic Research}

Case Rep Orthop Res 2019;2:61-68

DOI: $10.1159 / 000502733$

(C) 2019 The Author(s). Published by S. Karger AG, Basel www.karger.com/cio

Kishan et al.: Tuberculosis of the Hand with Isolated Hamate Involvement: An Unusual Presentation

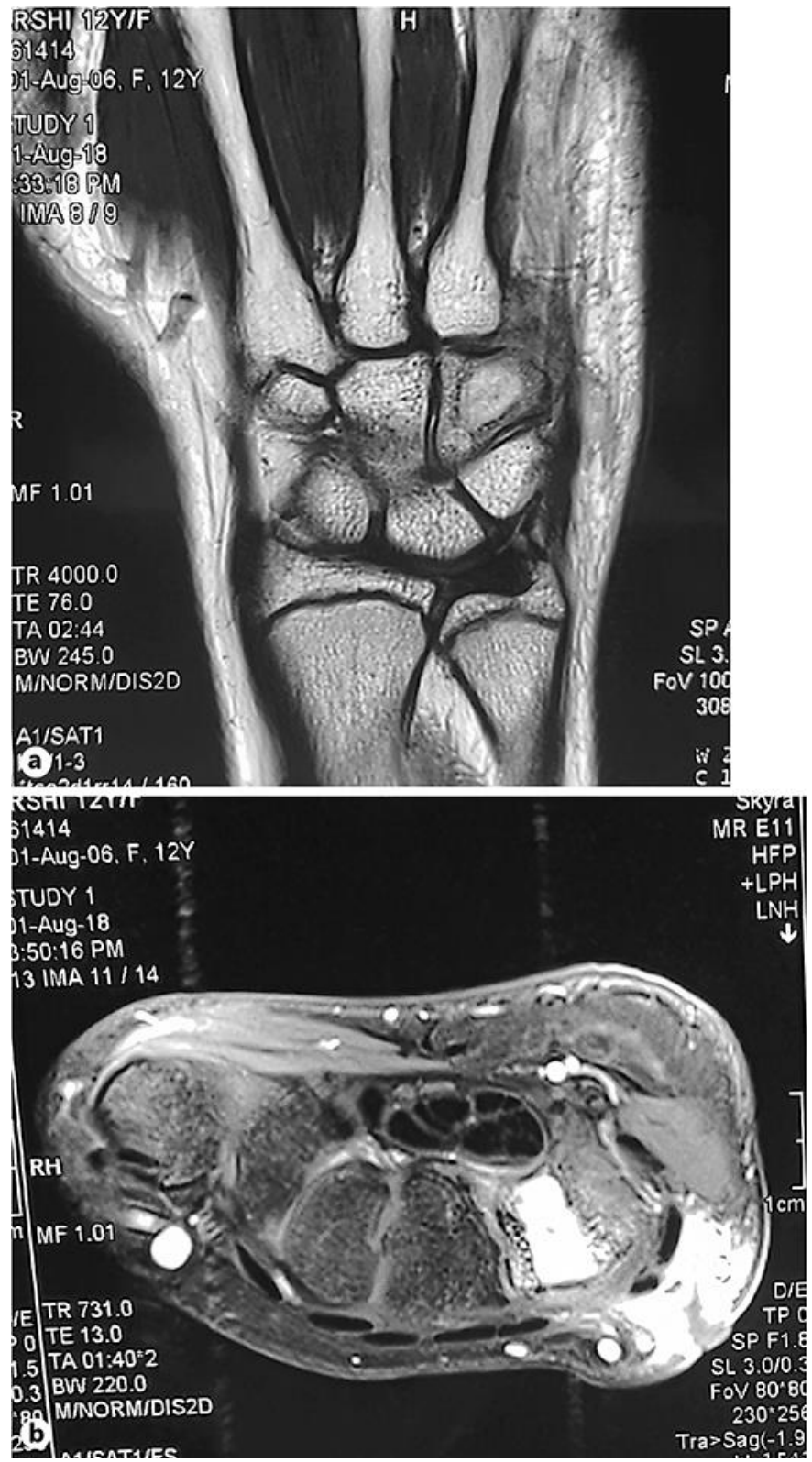

Fig. 2. a MRI of the wrist: T1-weighted image showing hypointense signal in the hamate. $\mathbf{b}$ MRI of the wrist: $\mathrm{T} 1$ with gadolinium contrast images showing hyperintensity with postcontrast enhancement. 


\section{Case Reports in Orthopedic Research}

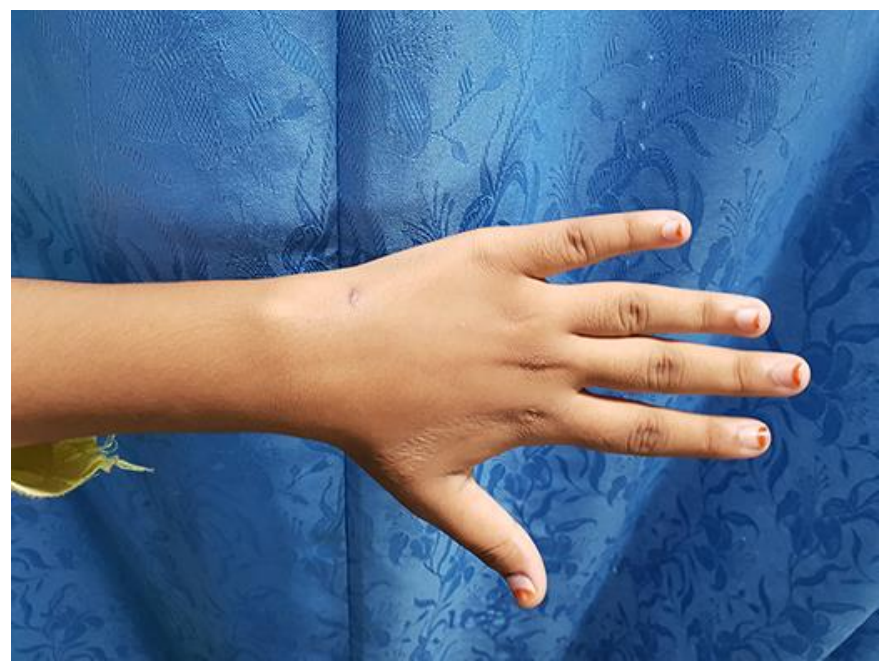

Fig. 3. Clinical picture after 6 months of antitubercular therapy.

Table 1. Isolated bone or joint involvement in wrist and hand TB

\begin{tabular}{llll}
\hline & Journal & Author & Site of involvement \\
\hline 2008 & Lung India & Kushwaha et al. [3] & 3rd metacarpal \\
2009 & Journal of Bone and Joint Surgery (Br.) & Kotwal et al. [18] & 6 cases of metacarpal \\
& & & TB, 2 cases of capitate \\
& & & TB, 2 cases of proximal \\
& & phalanx TB & Capitate \\
2014 & Malaysian Orthopaedic Journal & Sbai et al. [5] & Scapholunate joint \\
2015 & International Journal of Microbacteriology & Goel et al. [6] & Hamate osteitis \\
2015 & Journal of Clinical Orthopedics and Trauma & Prakash et al. [7] & Scaphoid \\
2015 & BMJ Case Report & Grenho et al. [8] & Capitate \\
2018 & BMJ Case Report & &
\end{tabular}

